\title{
FFAG R\&D activities in Japan and results from operating facilities
}

\section{Yoshiharu Mori ${ }^{1}$}

Kyoto University, Research Reactor Institute

Asashiro-nishi, Kumatori, Osaka, 590-0494, Japan

E-mail: morierri.kyoto-u.ac.jp

The recent activities on the development of FFAG accelerators in Japan are reviewed.

10th International Workshop on Neutrino Factories, Super beams and Beta beams Valencia, Spain

30 June - 05 July, 2008

Yoshiharu Mori $^{1}$ 


\section{Introduction}

The world's first proton FFAG accelerator (POP-FFAG) was built in Japan - at KEK - in 2000. At approximately the same time, it was recognized that FFAG accelerators may feature rapid acceleration with large momentum acceptance, exactly the properties required for both muon acceleration and production of proton beams for medical applications or for ADS (Accelerator Driven Systems for nuclear energy).

To investigate this potential, a prototype of large scale proton FFAG accelerator was developed at KEK. In 2004, it successfully accelerated a proton beam up to $150 \mathrm{MeV}$ with a repetition rate of $100 \mathrm{~Hz}$. Since then, intensive studies and discussions have taken place and various novel ideas have emerged which ultimately have led to new FFAG accelerator application projects at several institutes in Japan.

\section{FFAG R\&D activities in Japan}

\subsection{Kyoto University}

In Kyoto University, a proton FFAG accelerator has been developed for basic research work on ADS (Accelerator Driven System for nuclear energy) experiments. Here, the beam is delivered to the existing critical assembly (KUCA) of the Kyoto University Research Reactor Institute (KURRI). The whole machine is a cascade of three FFAG rings, as shown in Fig.1. Very recently, the beam was successfully accelerated up to $100 \mathrm{MeV}$. The first ADS experiment will start in the summer 2008. Kumatori accelerator driven Reactor Test (KART) project has been started at Kyoto University Research Reactor Institute (KURRI) from the fiscal year of 2002, aiming to demonstrate the basic feasibility of Accelerator Driven Sub-critical system (ADS) and to develop an $150 \mathrm{MeV}$ proton FFAG accelerator complex as a neutron, production driver[1]. The accelerator complex is composed three FFAG rings; injector, booster, and main ring[2]. The specifications of the each FFAG are summarized in Table 1. The beam was successfully accelerated up to $100 \mathrm{MeV}$ as shown in Fig. 2 in the main ring and will be extracted soon.

Table 1: Specification of FFAG complex for ADS at Kyoto University

\begin{tabular}{llll}
\hline & Injector & Booster & Main Ring \\
\hline Focusing & Spiral, 8 cells & Radial, 8 cells & Radial, 12 cells \\
Acceleration & Induction & RF & RF \\
Field index, $\mathrm{k}$ & 2.5 & 4.5 & 7.5 \\
Energy(Max) & $0.1-2.5 \mathrm{MeV}$ & $2.5-20 \mathrm{MeV}$ & $2-=150 \mathrm{MeV}$ \\
Pext/Pinj & $5.00(\max )$. & 2.84 & 2.83 \\
Orbit radius & $0.60-0.99 \mathrm{~m}$ & $1.42-1.71 \mathrm{~m}$ & $4.54-5.12 \mathrm{~m}$ \\
\hline
\end{tabular}


Medical applications of FFAG accelerators have also been proposed in two different fields: hadron therapy and boron neutron capture therapy (BNCT).[2]

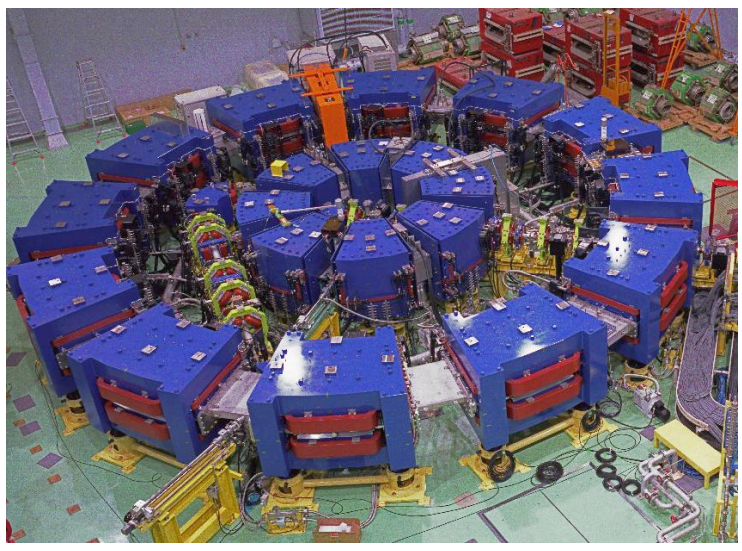

Fig. 1: Photograph of FFAG complex at Kyoto University Research Reactor Institute.

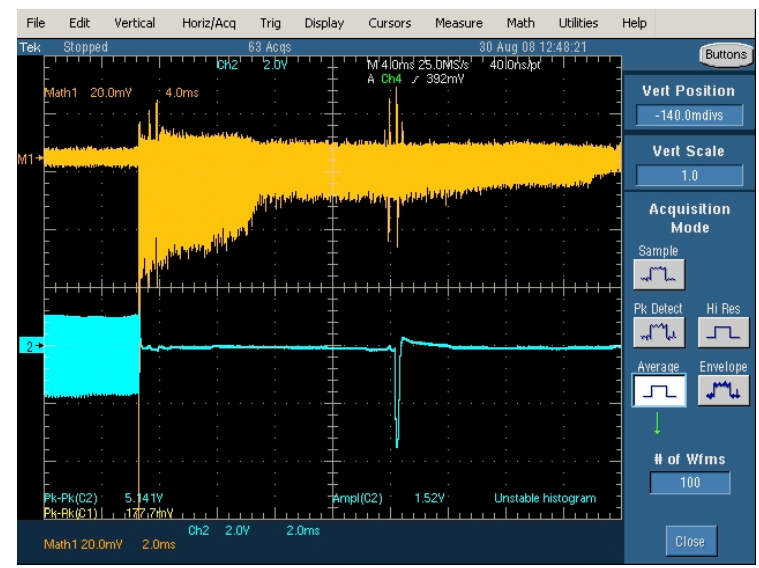

Fig. 2: Beam profile of the main ring.

For BNCT, an accelerator based intense thermal or epithermal neutron source has been developed at KURRI, using an FFAG storage ring with a thin internal Be target. The growth of the beam emittance and the energy distortion caused by scattering in the target can be controlled using ionization cooling, a functionality which could not be used in a cyclotron due to the lack of space. The whole system has been completed (Fig. 3) and recently the beam was successfully accumulated as expected in the ring. Neutron production has already been observed. This is the first experimental demonstration of the efficiency of ionization cooling.

\subsection{FFAG in Osaka University}

PRISM is an experimental proposal of the University of Osaka to build a highly-intense muon source using the $50 \mathrm{GeV}$ proton beam of the J-PARC synchrotron. In the PRISM project, longitudinal phase space rotation to narrow the initial energy spread of a muon beam by a scaling FFAG ring - featuring a large energy acceptance - has been developed to search for the 
lepton flavor violation in muon interactions. The ring consists of 10 magnets and 5 magnetic alloy RF cavities with a frequency and a gradient of $5 \mathrm{MHz}$ and $200 \mathrm{kV} / \mathrm{m}$, respectively.

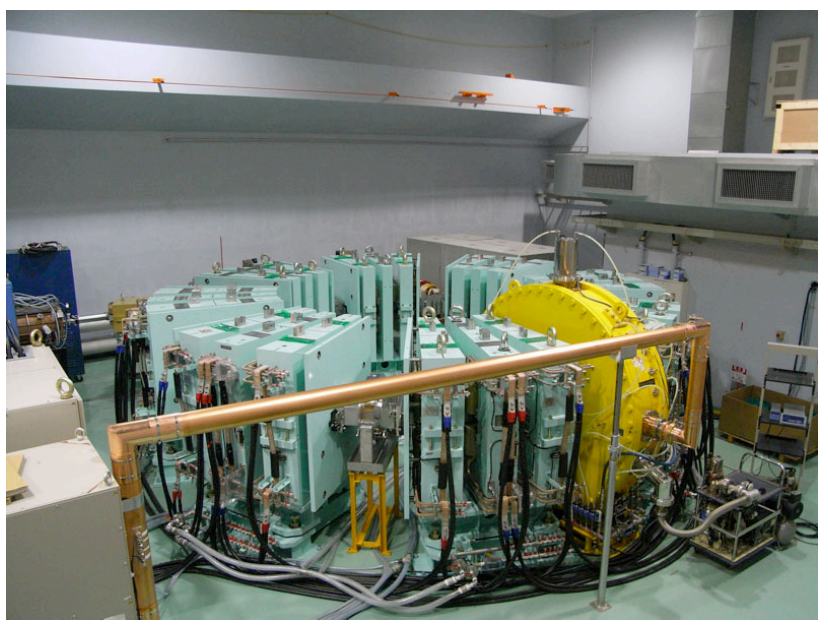

Fig. 3: Photograph of the neutron source with FFAG-ERIT based on ionization cooling.

\subsection{FFAG in Kyusyu Univeersity}

In the University of Kyusyu, a new accelerator facility for various applications, such as nuclear physics and material science, is under construction. The main machine will be a 150 $\mathrm{MeV}$ proton FFAG accelerator whose design closely follows the one of KEK as presented above.

\subsection{FFAG for industrial applications}

Electron Beam (EB) Accelerators have been used in the many industrial fields to improve physical properties of the material. Examples are wire and cable industries, rubber tire industries, foam industries, etc. EB is also widely used for medical device sterilization as a popular tool [3].

\begin{tabular}{ll}
\hline Energy & $50-500 \mathrm{keV}$ \\
\hline Cell number & 6 \\
\hline K value & 0.6 \\
\hline Orbit radius & $0.1900 .44 \mathrm{~m}$ \\
\hline Packing factor & 0.4 \\
\hline Spiral angle & $30 \mathrm{deg}$ \\
\hline Betatron tune & $1.3 / 1.2$ \\
\hline Repetition & $10 \mathrm{kHz}$ \\
\hline Duty factor & $20 \%$ \\
\hline Size (outer diameter) & $1.1 \mathrm{~m}$ \\
\hline
\end{tabular}

Table 2: Specifications of the prototype of electron FFAG. 
High power, high reliability, compactness and low cost are key requirements to get popularity of the technology and to open up its application fields. NHV Corporation has recently developed a compact electron FFAG for this purpose. The specifications of the electron FFAG are summarized in Table. 2. The beam acceleration in the accelerator is based on induction acceleration. In this case very long pulse beam can be accelerated, but the beam extraction from the FFAG ring is difficult normally. In this prototype FFAG accelerator we succeeded in the beam extraction from the FFAG ring using a combined septum system. Schematic layout of the accelerator is shown in Fig. 4

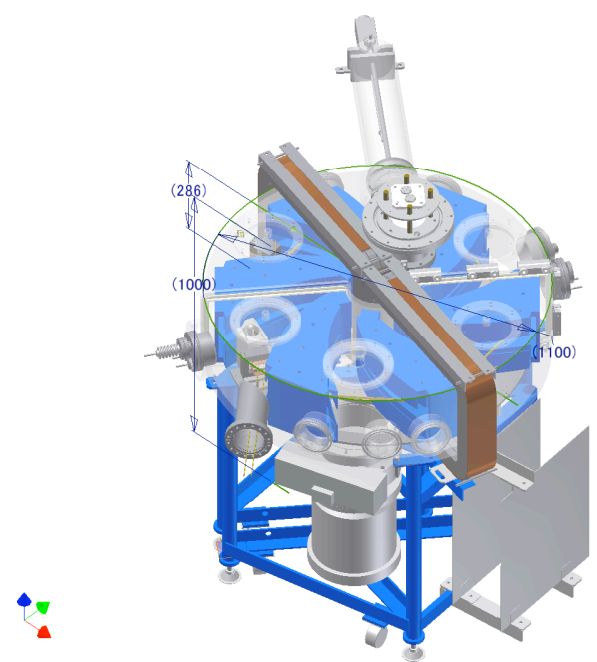

Fig. 4: Schematic layout of e-FFAG

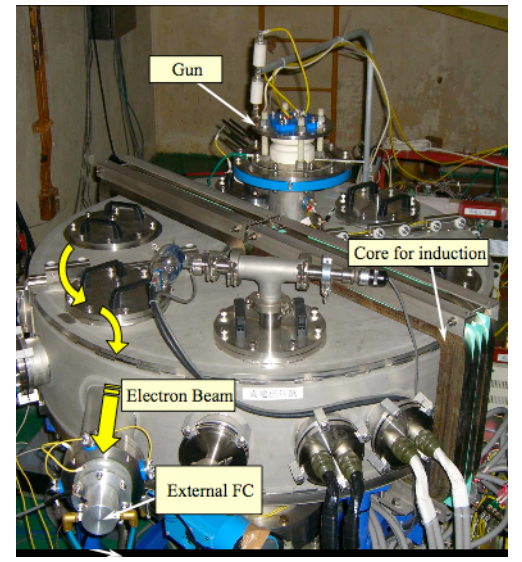

Fig. 5: Photograph of e-FFAG

Scheme of beam extraction is based on resonant extraction with a pair of massless and electrostatic septum magnets. More than $90 \%$ beam extraction efficiency was achieved. Figure 5 shows the beam profile of extracted beam measured by Faraday cup.

\section{Summary}

Recent R\&D activities of FFAG accelerators in Japan are briefly summarized.

\section{References}

[1] T. Uesugi et al., FFAGs for the ERIT and ADS projects at KURRI, in proceedings of EPAC08, TUOBM04,2008.

[2] K.Okabe et al., An intense neutron source with emittance recovery internal target (ERIT) using ionization cooling, in proceedings of EPAC08, THPP067, 2008.

[3] T. Baba, Development of FFAG electron accelrator, in proceedings of EPAC08, THPP001,2008. 